\title{
- Supplementary Material Scalable Approximate Bayesian Computation for Growing Network Models via Extrapolated and Sampled Summaries
}

\author{
Louis Raynal $^{*,}$, Sixing Chen ${ }^{*, \S}$, Antonietta Mira ${ }^{\dagger, \ddagger}$ and Jukka-Pekka Onnela*,
}

\section{Supplementary Analyses for the Simulation Study}

\subsection{Additional Covariance Functions for Gaussian Processes}

To complete the DMC simulation example, here we provide additional Gaussian process (GP) results when using the same mean function but employing different kernels. Our earlier choice was to use an additive combination of a linear kernel and a radial basis function (RBF) kernel. In addition to this sum-based version, we also study results using only the linear part, as well as a multiplicative combination of the linear and RBF kernels. When evaluated at $n_{1}$ and $n_{2}$ nodes, for the average degree (denoted $s_{1}$ ) and number of triangles (denoted $s_{2}$ ), the linear kernel is expressed as follows:

$$
\begin{aligned}
& \operatorname{Cov}\left(s_{1}\left(n_{1}\right), s_{1}\left(n_{2}\right)\right)=\left(\alpha \sqrt{n_{1}} \sqrt{n_{2}}+\gamma\right)+\sigma^{2} \mathbb{1}_{\left\{n_{1}=n_{2}\right\}}, \\
& \operatorname{Cov}\left(s_{2}\left(n_{1}\right), s_{2}\left(n_{2}\right)\right)=\left(\alpha n_{1} n_{2}+\gamma\right)+\sigma^{2} \mathbb{1}_{\left\{n_{1}=n_{2}\right\}},
\end{aligned}
$$

while for the linear times RBF kernel:

$$
\begin{aligned}
& \operatorname{Cov}\left(s_{1}\left(n_{1}\right), s_{1}\left(n_{2}\right)\right)=\left(\alpha \sqrt{n_{1}} \sqrt{n_{2}}+\gamma\right) \times \exp \left(-\frac{\left(n_{1}-n_{2}\right)^{2}}{2 \rho^{2}}\right)+\sigma^{2} \mathbb{1}_{\left\{n_{1}=n_{2}\right\}}, \\
& \operatorname{Cov}\left(s_{2}\left(n_{1}\right), s_{2}\left(n_{2}\right)\right)=\left(\alpha n_{1} n_{2}+\gamma\right) \times \exp \left(-\frac{\left(n_{1}-n_{2}\right)^{2}}{2 \rho^{2}}\right)+\sigma^{2} \mathbb{1}_{\left\{n_{1}=n_{2}\right\}},
\end{aligned}
$$

with $\alpha, \gamma, \rho, \sigma^{2}$ some positive parameters, specific to each kernel, and $\mathbb{1}$ being the indicator function. The prior for each hyper-parameter is again a standardized Gaussian distribution with identical truncation as specified in the main text. Moreover, we still consider the two possibilities to deduce the $\mathrm{ABC}$ posteriors, either using the densities of the reconstructed normal distributions (without inflated covariance matrices), or based on the distances between GP posterior means and observed summaries.

\footnotetext{
* Department of Biostatistics, T.H. Chan School of Public Health, Harvard University 655 Huntington Avenue, Building 2, 4th Floor, Boston, MA, USA 02115

${ }^{\dagger}$ Data Science Lab, Institute of Computational Science, Università della Svizzera italiana Via Buffi 6, 6900 Lugano, Switzerland

¥Dipartimento di Scienza e Alta Tecnologia, Università degli Studi dell’Insubria Via Valleggio, 11 - 22100 Como, Italy

$\S$ Shared first authors

๑ Corresponding author: llcraynal@hsph.harvard.edu
} 
The linear version is relevant as it provides covariance matrices similar to the ones determined empirically (Main Text, Figure 3), however it might lack flexibility. Regarding the linear times RBF kernel, it has the advantage of providing marginal variances that increase linearly with the number of nodes. It implies that the reconstructed normal distributions are less likely to all give zero density to the observed summary statistics, resulting in posteriors biased toward the prior means.

Figures 1, 2 and 3 show the bias as well as standard deviation (SD) and root mean squared error (RMSE) for the standard ABC (S-ABC) and the GP-based methods with the different kernels. These figures illustrate that the posterior means, using the linear kernel combined with density-based estimators, are always biased toward the prior means. This is explained by the GP posterior variances which are here extremely small. The distance-based version performs decently, though it is often outperformed by the linear plus RBF adaptation. The linear times RBF kernel shows great performance for all pair of parameters excepted the first one, for both density-based and distance-based methods, suggesting a large bias of the posterior GP mean. Finally, we can say that there is no specific kernel that outperforms all others for all true parameter pairs. This highlights the importance of selecting a flexible kernel, able to adjust to different shapes of tracked summaries, in different regions of the parameter space.

\subsection{Averaged Sample Triangle Counts}

Similarly to RE-ABC, which is subsampling 100 nodes to determine sample triangle count, we propose to reduce the variability of this summary by sampling it multiple times (10 or 20), and using the average value as a replacement for the triangle-based summary in the analysis. These are denoted 10 or 20 avg RE-ABC in the following. Excepted for this change of summary statistic, the extrapolation setting is the same as in the main text.

Figures 4, 5 and 6 show the bias, standard deviations (SD) as well as root mean squared errors (RMSE) for S-ABC and RE-ABC when summaries are tracked up to $n_{s}=500$ or $n_{s}=1000$ nodes. These figures highlight little differences in terms of posterior estimates when using either 1,10 or 20 replicates. Such similarity can be explained by very similar fits between the least-squares adjusted polynomial functions for the sample triangle count, and thus for the extrapolated summary values as well. As expected, we again observe a small gain when tracking up to $n_{s}=1000$ nodes compared to 500 . 

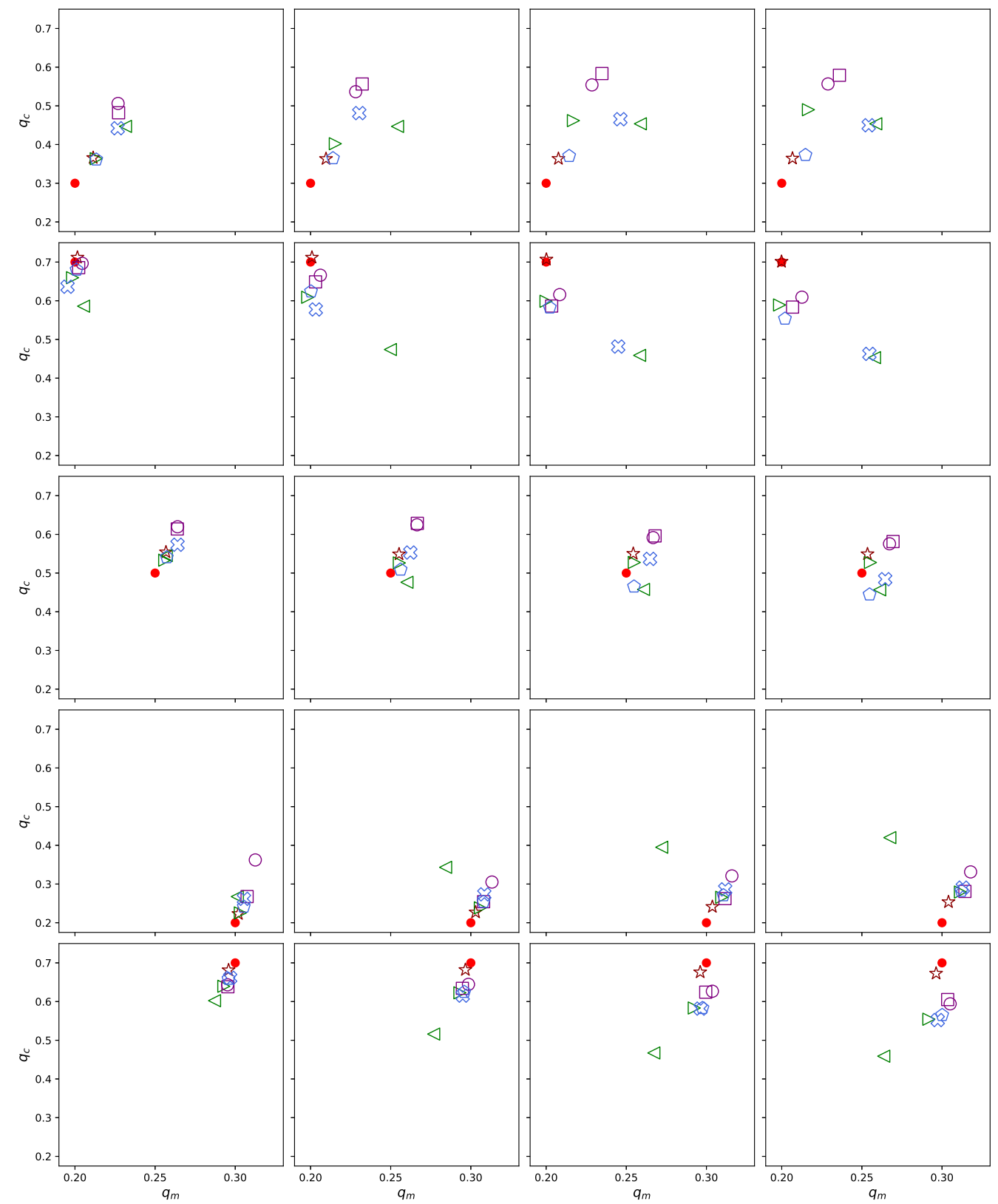

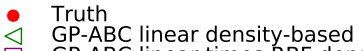

GP-ABC linear times RBF density-based

GP-ABC linear plus RBF density-based

St) $\mathrm{S}-\mathrm{ABC}$

G GP-ABC linear distance-based

GP-ABC linear plus RBF distance-based

Figure 1: Average posterior means provided by the standard $\mathrm{ABC}$ (S-ABC), and the GP-based methods with the different kernel functions. The red dot denotes the true parameter value. Each row corresponds to different values of the true parameter $\left(q_{m}, q_{c}\right) \in\{(0.2,0.3),(0.2,0.7),(0.25,0.5),(0.3,0.2),(0.3,0.7)\}$, each column corresponds (from left to right) to values of $n_{o}=1000,2000,5000,10000$. 

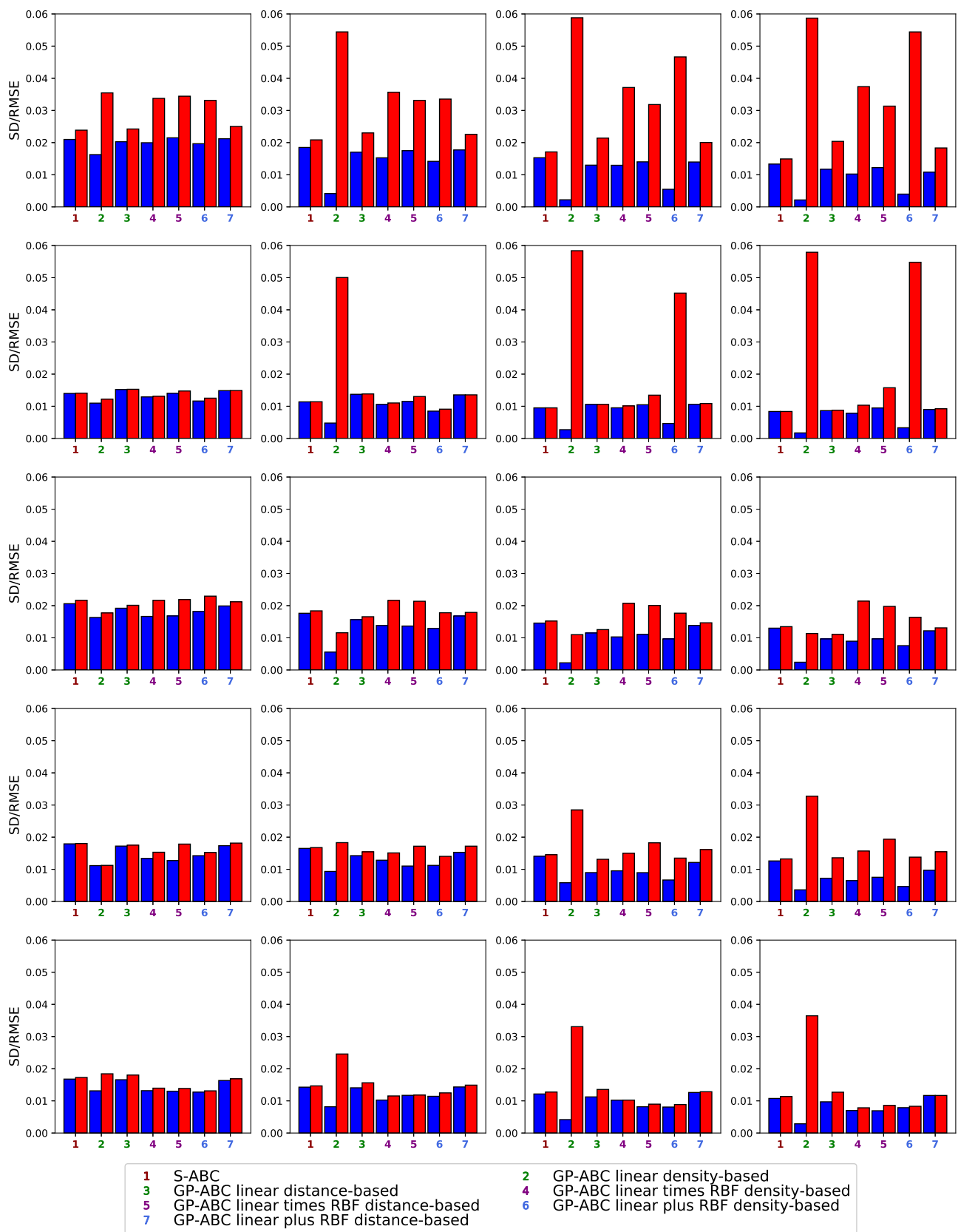

Figure 2: Standard deviation (SD, left bar of each pair) and root mean square error (RMSE, right bar of each pair) of the different estimators employing GPs for $q_{m}$. Each row corresponds to different values of the true parameter $\left(q_{m}, q_{c}\right) \in\{(0.2,0.3),(0.2,0.7)$, $(0.25,0.5),(0.3,0.2),(0.3,0.7)\}$, each column corresponds (from left to right) to values of $n_{o}=1000,2000,5000,10000$. 

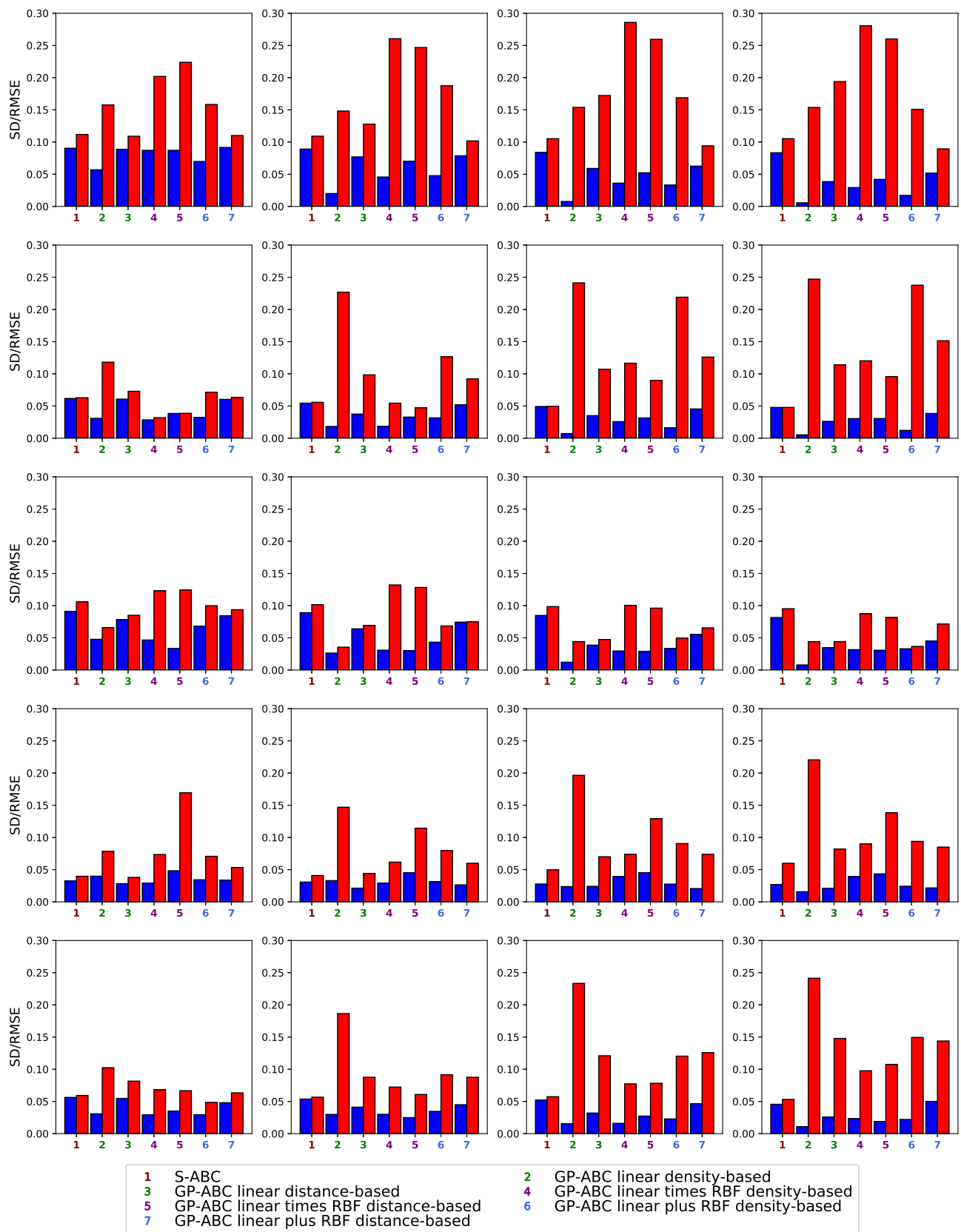

Figure 3: Standard deviation (SD, left bar of each pair) and root mean square error (RMSE, right bar of each pair) of the different estimators employing GPs for $q_{c}$. Each row corresponds to different values of the true parameter $\left(q_{m}, q_{c}\right) \in\{(0.2,0.3),(0.2,0.7)$, $(0.25,0.5),(0.3,0.2),(0.3,0.7)\}$, each column corresponds (from left to right) to values of $n_{o}=1000,2000,5000,10000$. 


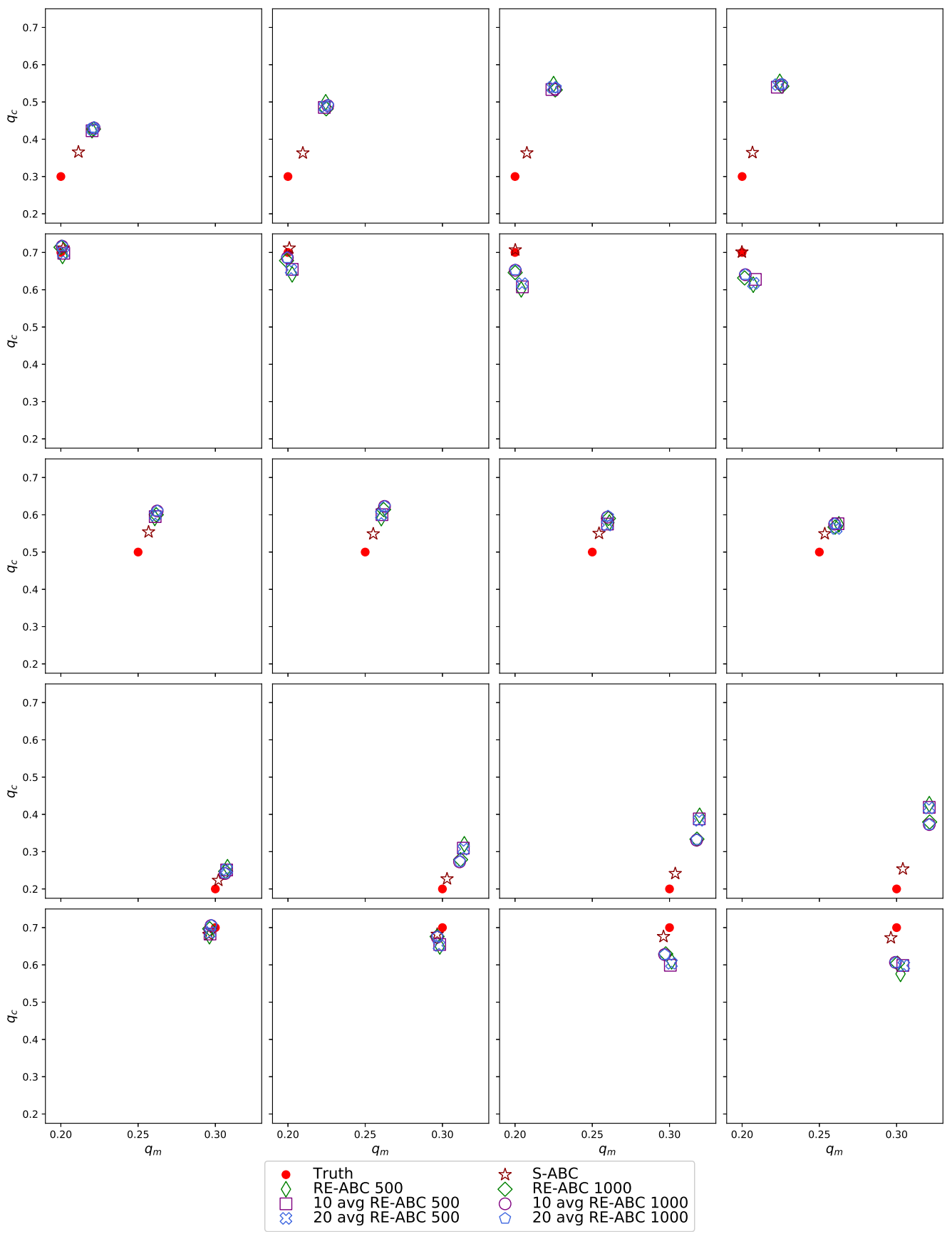

Figure 4: Average posterior means provided by the standard ABC (S-ABC), and leastsquares based methods when employing a single sample triangle count, or the averaged sample triangle count obtained over 10 or 20 replicates. The red dot denotes the true parameter value. Each row corresponds to different values of the true parameter $\left(q_{m}, q_{c}\right) \in\{(0.2,0.3),(0.2,0.7),(0.25,0.5),(0.3,0.2),(0.3,0.7)\}$, each column corresponds (from left to right) to values of $n_{o}=1000,2000,5000,10000$. 

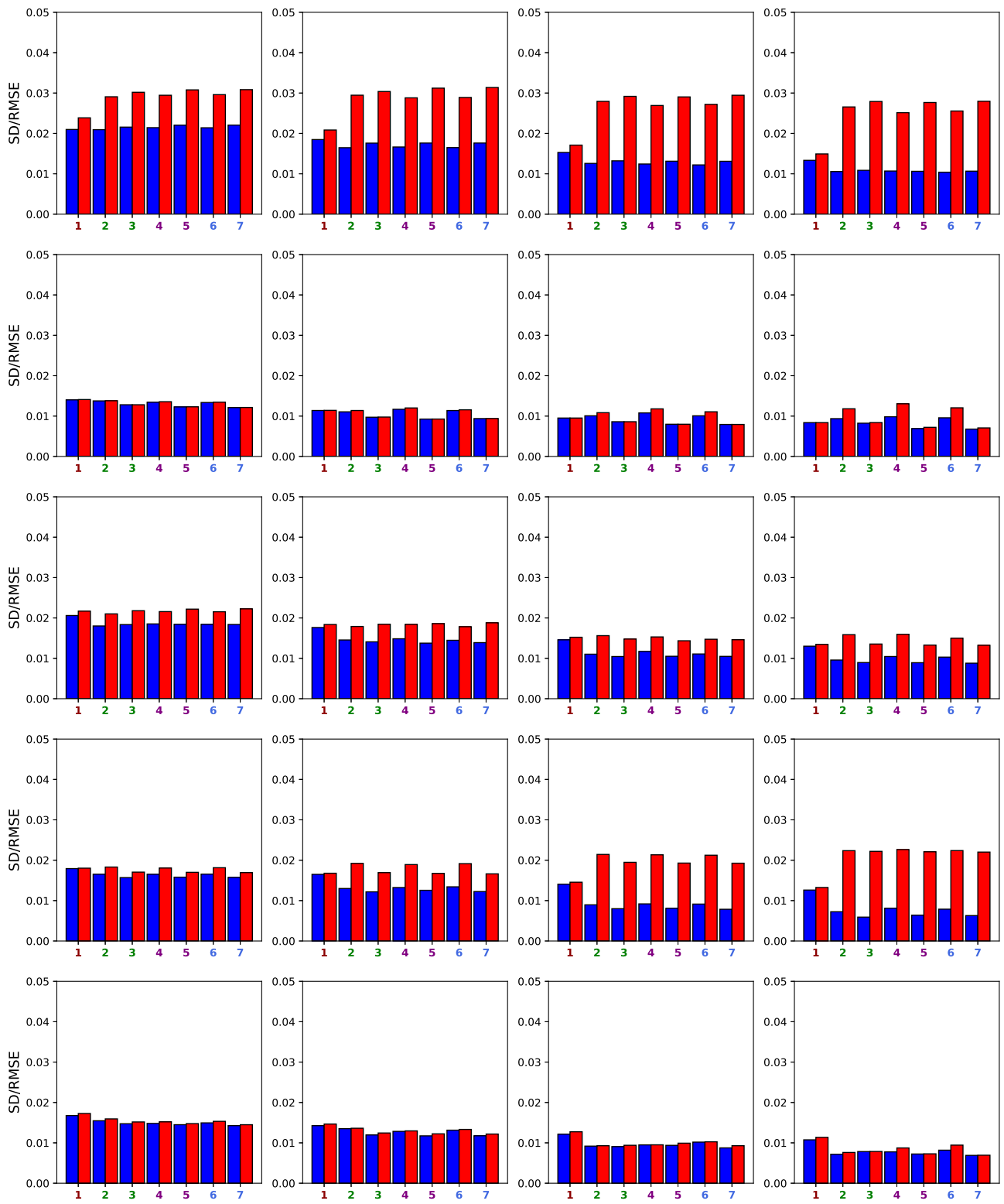

1 S-ABC

RE-ABC 1000

10 avg RE-ABC 1000

2 RE-ABC 500

$4 \quad 10$ avg RE-ABC 500

720 avg RE-ABC 1000

Figure 5: Standard deviation (SD, left bar of each pair) and root mean square error (RMSE, right bar of each pair) of the different estimators for $q_{m}$. Each row corresponds to different values of the true parameter $\left(q_{m}, q_{c}\right) \in\{(0.2,0.3),(0.2,0.7),(0.25,0.5)$, $(0.3,0.2),(0.3,0.7)\}$, each column corresponds (from left to right) to values of $n_{o}=$ $1000,2000,5000,10000$. 

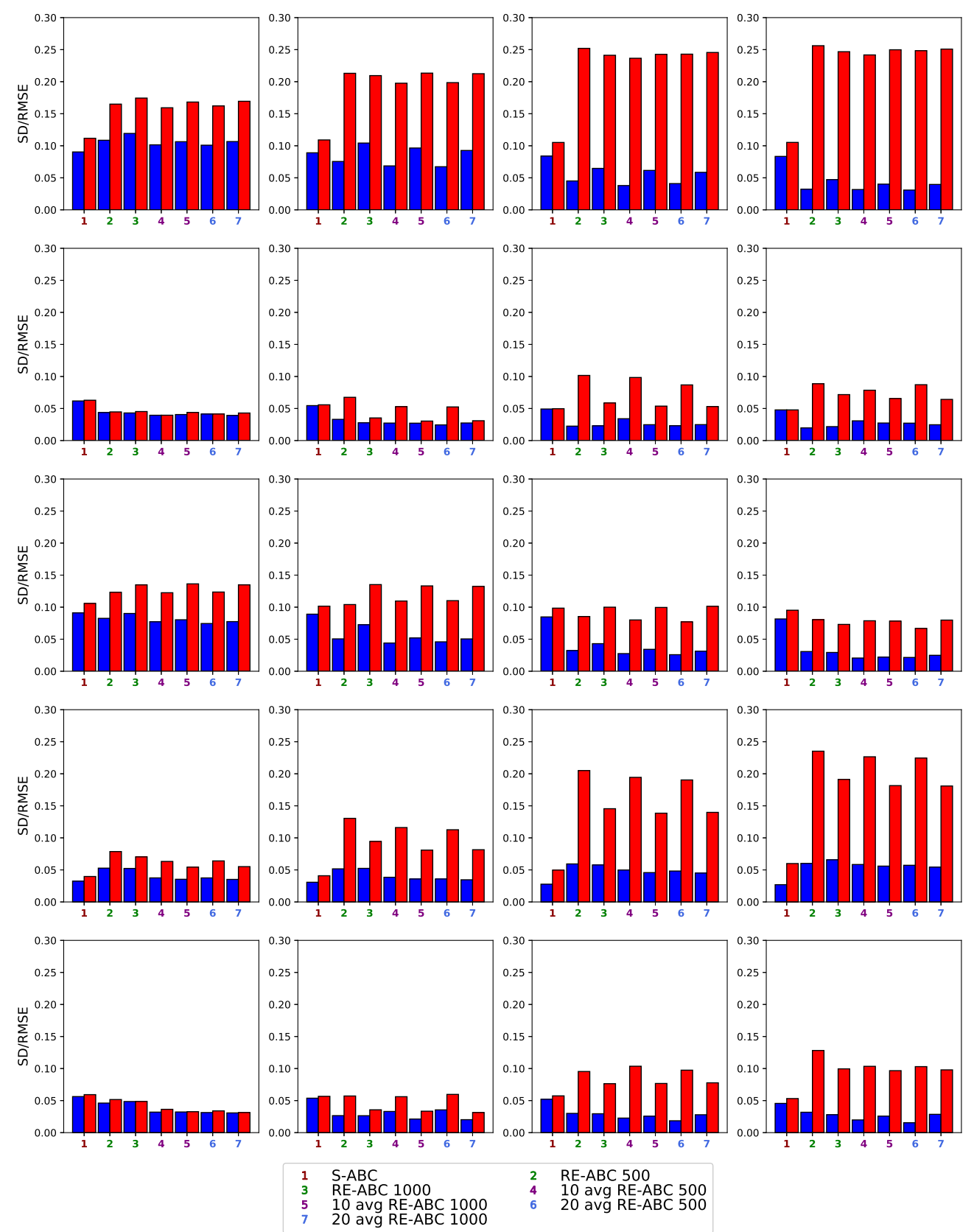

Figure 6: Standard deviation (SD, left bar of each pair) and root mean square error (RMSE, right bar of each pair) of the different estimators for $q_{c}$. Each row corresponds to different values of the true parameter $\left(q_{m}, q_{c}\right) \in\{(0.2,0.3),(0.2,0.7),(0.25,0.5)$, $(0.3,0.2),(0.3,0.7)\}$, each column corresponds (from left to right) to values of $n_{o}=$ 1000, 2000, 5000, 10000 . 